\title{
Pyramid Mekaniska Konsult (PMK) HB
}

\section{Giottis Motsanos*}

Trifilon Technologies, Sweden

Pyramid Mekaniska Konsult (PMK) has defined the problems referable to the "Golden Role of Mechanics" which rule stipulates "What is gained in power is lost in working distance" by using two independent axels in the closed "inertial" system. The EME system separates the two variables in the pulley system.

- The dependency variable - power,

- $\quad$ The independent variable - working distance (Figure 1).

By achieving the above, the outgoing axel "power" has the same speed as the ingoing axel "working distance". Conclusion: We can reduce the working distance in the ingoing axel of the motor without losing power and speed on the outgoing axel of the generator. This is achieved by synchronising and harmonising the system by a theory of mathematics. Pyramid Mekaniska Konsult (PMK) has a high standard of scientific method which can not be disputed. In my opinion the theory and emperical data can not be falsified, only be confirmed by any party whishing to do so. It is the same situation as that of the Higgs partical.

\section{The EME System}

The unique thing about the EME system is that it allows for using all the fundamental forces to it's advantage and quantum phenomena in a closed "inertial" system such as shown to the left respecively to the right below (Figure 2).

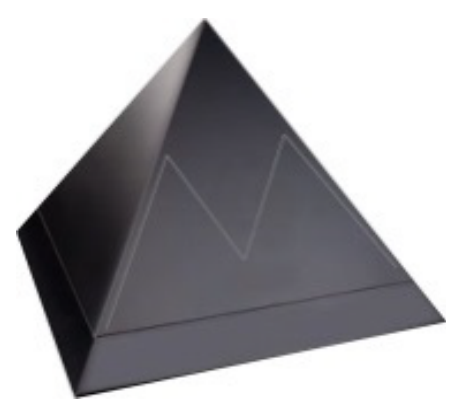

Figure 1: Pyramid Mekaniska Konsult HB.
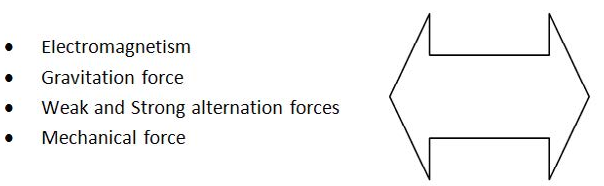

Quantum entangelment Quantum superposition Spinn

- Mechanical force

Figure 2: EME system.

The emperical data that Pyramid Mekaniska Konsult (PMK) has produced goes hand in hand with the law of physics and quantum physics and can be supported and acknowledged by Planck, Feynman, Dirac and foremost

\section{Hendrik Lorentz as it is a copy of his theory}

In my platform of calculations I am using scientiests such as: Newton, Arkimedes, Einstein, Gauss and Pythagoras. Pyramid Mekaniska Konsult (PMK) has also taken in consideration, Bohr's

Unpredictability and Heisenberg's Uncertainty Principal.

The string theory works as a bridge between "The general theory of relativity" and the mechanics. Many scientists state that the string theory is a bridge between quantum mechanics and "The general theory of relativity" only, which is wrong in my opinion and $\mathrm{Mr}$. Andrej Sacharov supports my opinion. Pyramid Mekaniska Konsult (PMK) is able to incorporate gravity in its theory and functionality which quantum mechanics can not, as well as using Weak and Strong alternation forces which quantum mechanics cannot, in the same way or energy output.

A paradigm shift is at hand and that is not a moment too soon in order to get new knowledge and to save the world from greenhouse gases. It is easy to be a no-teller, everyone is welcome to validate test data and see if the theory and reality are holding a scientific review. Only a tough scrutiny from a independent source will increase PMK:s crediability. Following the Copenhagen interpretation, nothing can be said about quantum physical systems and phenomena until emperical data has been produced.

The uniqueness of PMK:s invention is the ability to think outside the box, in other words simplicity is the ultimate sophistication. Mathematical calculations and theories can be obtained when an NDA and cooperation agreement has been signed by both parties.

Copy right @: PMK HB 2014.
*Corresponding author: Motsanos G, Founder, President at PMK and Marketing Director, Trifilon Technologies, Sweden, Tel: +46 8162000; E-mail: g.motsanos@hotmail.com

Received July 19, 2017; Accepted August 08, 2017; Published August 15, 2017

Citation: Motsanos G (2017) Pyramid Mekaniska Konsult (PMK) HB. Fluid Mech Open Acc 4: 170. doi: 10.4172/2476-2296.1000170

Copyright: (C) 2017 Motsanos G. This is an open-access article distributed under the terms of the Creative Commons Attribution License, which permits unrestricted use, distribution, and reproduction in any medium, provided the original author and source are credited. 